\title{
Erratum to: Negative feedback of miR-29 family TET1 involves in hepatocellular cancer
}

\author{
Li Li Lin · Wei Wang · ZhaoYang Hu • \\ Li Wen Wang · Jing Chang $\cdot$ HanGuang Qian
}

Published online: 24 January 2015

(c) Springer Science+Business Media New York 2015

\begin{abstract}
Primary hepatocellular carcinoma (HCC) is the most common form of liver cancer and is one of the most common malignancies worldwide. Tumor suppressor gene silencing through DNA methylation contributes to cancer formation. The ten-eleven translocations (TET) family of $\alpha$-ketogluta-rate-dependent dioxygenases catalyzes the sequential oxidation of 5-methylcytosine to 5-hydroxymethyl-cytosine, 5-formylcytosine and 5-carboxylcytosine, leading to eventual DNA demethylation. MicroRNAs are an abundant class of 17-25 nucleotides small noncoding RNAs, identified as important regulators of many diverse biological processes. In this study, we showed that TET1 expression was obviously reduced in the majority of examined HCC tissues. And we further investigated the expression and functional involvement of TET1 in
\end{abstract}

Li Li Lin and Wei Wang have contributed equally to this work.

The online version of the original article can be found under doi:10.1007/s12032-014-0291-2.

Erratum to: Med Oncol (2014) 31:291. DOI 10.1007/s12032-0140291-2

Due to errors in the experimental results section, this article is being reprinted in its entirety. This version contains the corrected experimental results.

L. L. Lin · L. W. Wang · J. Chang

Department of Pharmacology, Wuxi Higher Health Vocational

Technology School, No. 305, Xinguang Road, Wuxi 214028,

China

W. Wang

Department of Pharmacy and Medical Appliances, Hangzhou Sanatorium of PLA, No. 27 Yang Gong Dike,

Hangzhou 310007, Zhejiang, China proliferation, migration and invasion and determined that TET1 may function as a tumor suppressor. miR-29b was proved to inhibit metastasis through the targeting of TET1, indicating that downregulation of miR-29 may involve in HCC carcinogenesis and progression through potentiation of TET1 expression. Thus, we elucidated the roles of feedback of miR-29-TET1 downregulation in HCC development and suggested a potential target in identification of the prognosis and application of cancer therapy for HCC patients.

Keywords miR-29 family TET1 · Hepatocellular cancer . miRNAs $\cdot$ Negative feedback

$\begin{array}{ll}\text { Abbreviations } \\ \text { HCC } & \text { Primary hepatocellular carcinoma } \\ \text { TET } & \text { Ten-eleven translocation } \\ \alpha-\text { KG } & \alpha \text {-Ketogluta-rate } \\ \text { 5mC } & \text { 5-Methylcytosine } \\ \text { 5hmC } & \text { 5-Hydroxymethyl-cytosine } \\ \text { miRNAs } & \text { MicroRNAs } \\ \text { DMEM } & \text { Dulbecco's modified Eagle's medium } \\ \text { HH } & \text { Human hepatocytes } \\ \text { TCL1 } & \text { T cell leukemia/lymphoma 1 } \\ \text { Mcl-1 } & \text { Myeloid cell leukemia sequence 1 } \\ \text { CDC42 } & \text { Cell division cycle 42 }\end{array}$

Z. Hu

Cancer Research Institute, Hangzhou Cancer Hospital, No. 34 Yan Guan Road, Hangzhou 310002, China

H. Qian $(\bowtie)$

Department of Orthopedics, Wuxi Third People's Hospital Affiliated, School of Medicine, Nantong University, No. 585 Northern Xinyuan Rd, Wuxi 214041, China e-mail: qianhanguang0419@126.com 
EGFP Enhanced green fluorescent protein

FBS

\section{Introduction}

Primary hepatocellular carcinoma (HCC) is the most common form of liver cancer and one of the most common malignancies worldwide, with nearly 600,000 deaths each year [1, 2]. In China, the incidence of HCC is increasing and $\mathrm{HCC}$ becomes the second most common cause of cancer mortality despite therapeutic advances. However, the molecular mechanisms underlying pathogenesis of $\mathrm{HCC}$ remain obscure.

Tumor suppressor gene silencing through DNA methylation contributes to cancer formation. The ten-eleven translocation (TET) family of $\alpha$-ketogluta-rate $(\alpha-\mathrm{KG})$ dependent dioxygenases catalyzes the sequential oxidation of 5-methylcytosine $(5 \mathrm{mC})$ to 5-hydroxymethylcytosine $(5 \mathrm{hmC}), 5$-formylcytosine and 5-carboxylcytosine, leading to eventual DNA demethylation. TET protein family contains three members (Tet1, Tet2 and Tet3). TET1 was the first to be identified as the gene that was fused to the MLL gene in certain leukemia patients due to a chromosome translocation [3]. Chih-Hung Hsu has found that a dioxygenase involved in cytosine demethylation is downregulated in prostate and breast cancer tissues. TET1 depletion facilitates cell invasion, tumor growth and cancer metastasis in prostate xenograft models and correlates with poor survival rates in breast cancer patients [4]. The TET2, which was recently discovered, has drawn most attention, and it has been identified as one of the most frequently mutated genes in myeloid malignancies and other types of cancers [5-7]. Whereas some of the mutations sited in TET2 correspond to residues within the catalytic domain and could thus impair catalytic activity, many mutations appear unrelated to enzymatic activity [8]. In theory, the molecular mechanism underlying TET in cancer is still unclear and needs further studies.

MicroRNAs (miRNAs) are an abundant class of 17-25 nucleotides small noncoding RNAs, identified as important regulators of many diverse biological processes. It has been found that miRNAs could regulate protein-coding genes expression at the post-transcriptional level through binding to the $3^{\prime}$ untranslational region ( $3^{\prime} \mathrm{UTR}$ ) of target mRNAs [9]. By regulating the expression of target genes, miRNAs are involved in a variety of biological processes including cell cycle regulation, differentiation, development, metabolism, neuronal patterning and aging [10]. After initial observation, about 1,000 miRNA sequences have been identified in mammals, but the biological roles in tumorigenesis of remain elusive in a large part. A growing body of evidence has demonstrated that miRNAs have played important roles in cancer development and clinical outcomes of cancer patients [11]. Up to date, different study cohorts find that miRNAs are involved in HCC development and some of them have been identified to correlate with prognosis or accepted to be potential therapeutic targets [12-15]. For example, the study by Jiang and his colleagues revealed that miR-199a, miR-21 and miR-301 were differentially expressed between tumor and adjacent benign liver. On the other hand, a large number of mature and precursor miRNAs were upregulated in the adjacent benign liver specimens [16]. Furthermore, several deregulated miRNAs (e.g., miR-21, miR-101, miR-195, miR-122, miR-221, miR-223 and miR-224) have been shown to regulate cell growth, apoptosis, migration or invasion [1722]. In conclusion, the ability of miRNAs to function as tumor promoters or suppressors in hepatocarcinogenesis has led to new insights into the molecular pathways involved in HCC [14, 23]. To date, several oncogenes, caused by apoptosis-related molecules, such as $\mathrm{T}$ cell leukemia/lymphoma 1 (TCL1), myeloid cell leukemia sequence 1 (Mcl-1), cell division cycle 42 (CDC42) and phosphoinositide-3-kinase regulatory subunit 1 (PIK3R1), have been characterized as targets of the miR family [2429]. However, miR-29 disfunction in hepatocarcinogenesis and the signaling pathways by which miR-29 exerts its function and modulates the malignant phenotypes of HCC cells remain largely unknown.

In this study, we showed that TET1 expression was obviously reduced in the majority of examined $\mathrm{HCC}$ tissues. We further investigated the expression and functional involvement of TET1 in proliferation, migration and invasion. We decided that TET1 might function as a tumor suppressor and MiR-29b inhibit metastasis through the targeting of TET1, which indicated that downregulation of miR-29 might be involved in HCC carcinogenesis and progression through potentiation of TET1 expression. Thus, we elucidated feedback of miR29-TET1 downregulation in HCC development. We suggested miR-29-TET1 be a potential target in identification of the prognosis and application of cancer therapy for HCC patients.

\section{Materials and methods patients and tissue samples}

Surgical resection paired HCC and adjacent non-neoplastic tissues were obtained from 25 patients at Wuxi Third People's Hospital (Wuxi, China) after surgical resection with informed consent. And the details are shown in Table 1. The tumor tissues and the adjacent normal tissues 
Table 1 Clinical data of 25 patients with HCC

\begin{tabular}{|c|c|c|c|c|c|c|c|c|c|c|}
\hline $\begin{array}{l}\text { Patient's } \\
\text { number }\end{array}$ & Sex & $\begin{array}{l}\text { Age } \\
\text { (years) }\end{array}$ & $\begin{array}{l}\text { Tumor } \\
\text { grade }\end{array}$ & Cirrhosis & HBsAg & $\begin{array}{l}\text { AFP } \\
(\mu \mathrm{g} / \mathrm{l})\end{array}$ & $\begin{array}{l}\text { CEA } \\
(\mu \mathrm{g} / \mathrm{l})\end{array}$ & $\begin{array}{l}\text { CA19-9 } \\
\text { (Units/ml) }\end{array}$ & $\begin{array}{l}\text { ALT } \\
\text { (Units/ml) }\end{array}$ & $\begin{array}{l}\text { AST } \\
\text { (Units/ml) }\end{array}$ \\
\hline 1 & M & 48 & 3 & - & + & 540 & 112 & 150.1 & 3,500 & 590 \\
\hline 2 & M & 64 & 3 & - & - & 620 & 124 & 14.7 & 202 & 38 \\
\hline 3 & M & 43 & 3 & - & + & 14,000 & 478 & 460 & 780 & 67 \\
\hline 4 & M & 62 & 2 & - & + & 4.6 & 2.8 & 4.8 & 455 & 89 \\
\hline 5 & M & 76 & 3 & - & + & 460 & 13 & 2.1 & 3,471 & 102 \\
\hline 6 & M & 66 & 4 & - & + & 260 & 8.3 & 4.5 & 891 & 1349 \\
\hline 7 & M & 48 & 3 & - & + & 361.2 & 1.2 & 46.4 & 824 & 394 \\
\hline 8 & M & 37 & 4 & - & + & 1,000 & 2.2 & 8.4 & 384 & 300 \\
\hline 9 & M & 16 & 1 & - & - & 22.6 & 5.4 & 3.2 & 121 & 78 \\
\hline 10 & M & 44 & 3 & + & + & 400 & 7.7 & 5.2 & 880 & $231 /$ \\
\hline 11 & $\mathrm{~F}$ & 33 & 3 & + & + & 30,000 & 6.1 & 45 & 74 & 58 \\
\hline 12 & M & 44 & 3 & + & + & 23.4 & 6.4 & 2.1 & 108 & 80 \\
\hline 13 & M & 55 & 3 & + & + & 21.1 & 9.0 & 4.5 & 57 & 107 \\
\hline 14 & M & 60 & 3 & + & - & 16.7 & 3.2 & 7.6 & 32 & 231 \\
\hline 15 & M & 50 & 3 & + & + & 18.2 & 3.6 & 2.1 & 113 & 440 \\
\hline 16 & $\mathrm{~F}$ & 61 & 3 & - & + & 1,233 & 143 & 692 & 17 & 18 \\
\hline 17 & $\mathrm{~F}$ & 61 & 4 & - & - & 13.1 & 11 & 3.4 & 78 & 41 \\
\hline 18 & $\mathrm{M}$ & 64 & 3 & - & + & 312 & 0.8 & 6.2 & 111 & 45 \\
\hline 19 & M & 51 & 3 & + & + & 24.3 & 3.5 & 2.1 & 104 & 424 \\
\hline 20 & M & 49 & 3 & + & + & 1,000 & 7.7 & 63 & 88 & 319 \\
\hline 21 & M & 39 & 3 & + & + & 131 & 7.1 & 67 & 55 & 85 \\
\hline 22 & $\mathrm{M}$ & 46 & 3 & + & + & 1,642 & 2.4 & 109 & 203 & 302 \\
\hline 23 & $\mathrm{M}$ & 51 & 3 & + & + & 189 & 14 & 32 & 89 & 37 \\
\hline 24 & M & 63 & 3 & - & 0 & 21.1 & 12 & 3.1 & 30 & $42 /$ \\
\hline 25 & $\mathrm{M}$ & 55 & 3 & + & + & 1,137 & 57 & 78 & 46 & 69 \\
\hline
\end{tabular}

$M$ male, $F$ female, AFP alpha fetoprotein, CEA carcinoembryonic antigen, CA19-9 carbohydrate antigen 19-9, AST aspartate aminotransferase, $A L T$ alanineaminotransferase

were quickly frozen in liquid nitrogen after resection until analysis. No patient in the current study received chemotherapy or radiation therapy before the surgery. This study was performed with the approval of the Wuxi Third People's Hospital and was conducted according to the Declaration of Helsinki Principles.

\section{Cell lines and culture}

HepG2 and Huh-7 human HCC cells were maintained in Dulbecco's modified Eagle's medium (DMEM) (GIBCO, Gaithersburg, MD). Normal human hepatocytes (HH) were obtained from Sciencell (San Diego, CA) and cultured following the manufacturer's instructions.

RNA extraction and quantitative RT-PCR

Total RNA, including miRNA, was extracted using TRIzol reagent (Invitrogen, Carlsbad, CA, USA) according to the manufacturer's instructions. qRT-PCR was used to confirm the expression levels of mRNAs and miRNAs as described before. U6 snRNA and G3PDH mRNA levels were used for normalization.

RNA Oligoribonucleotides and Plasmids

All RNA oligoribonucleotides were purchased from GenePharma. miRNA duplexes corresponding to mature miR-29a, miR-29b and miR-29c were designed as described [27]. The small interfering RNAs that targeted human TET1 mRNAs were designated siTET1. The negative control RNA duplex (named NC) for both miRNAmimic and small interfering RNA was non-homologous to any human genome sequences. The anti-miR-29a, anti-miR$29 \mathrm{~b}$ and anti-miR-29c, with sequences that were complementary to the mature miR-29a, miR-29b and miR-29c, respectively, were $2^{\prime}$ - $O$-methyl-modified oligoribonucleotides designed as inhibitors of miR-29a, miR-29b and miR$29 \mathrm{c}$ individually. The anti-miR-C was used as a negative control in the antagonism experiments. 
A

B
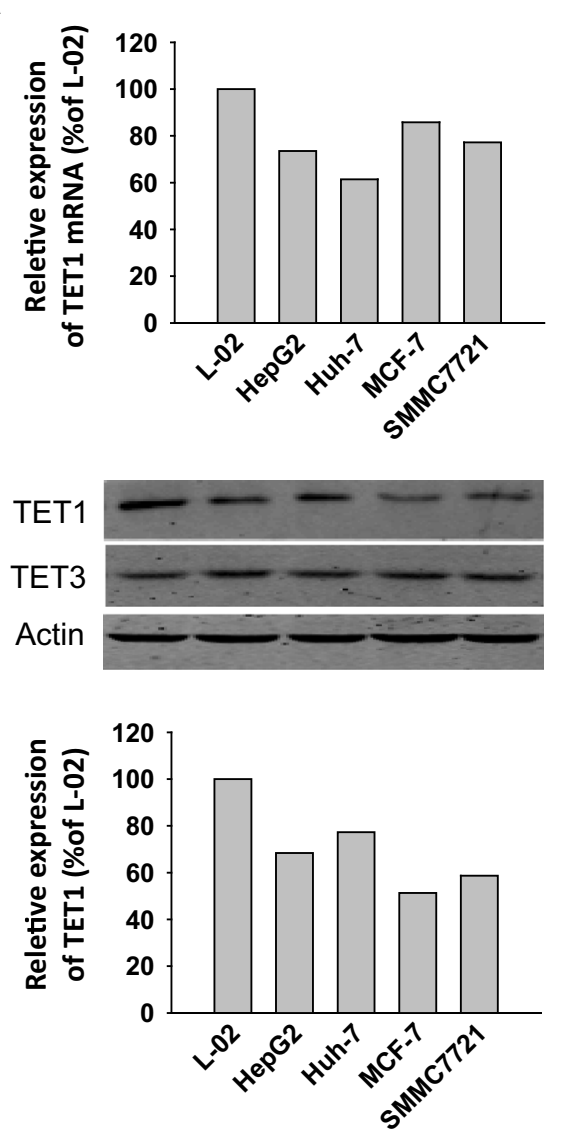

c
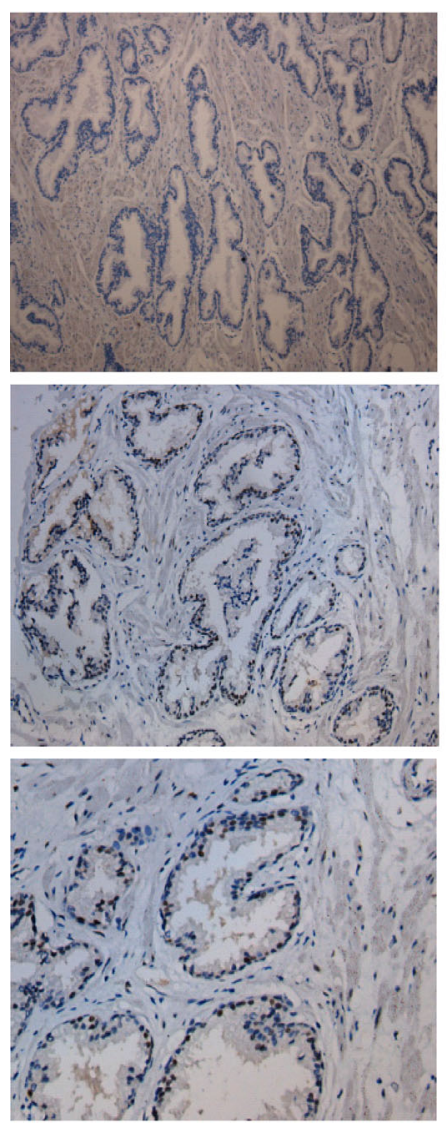

Cancer
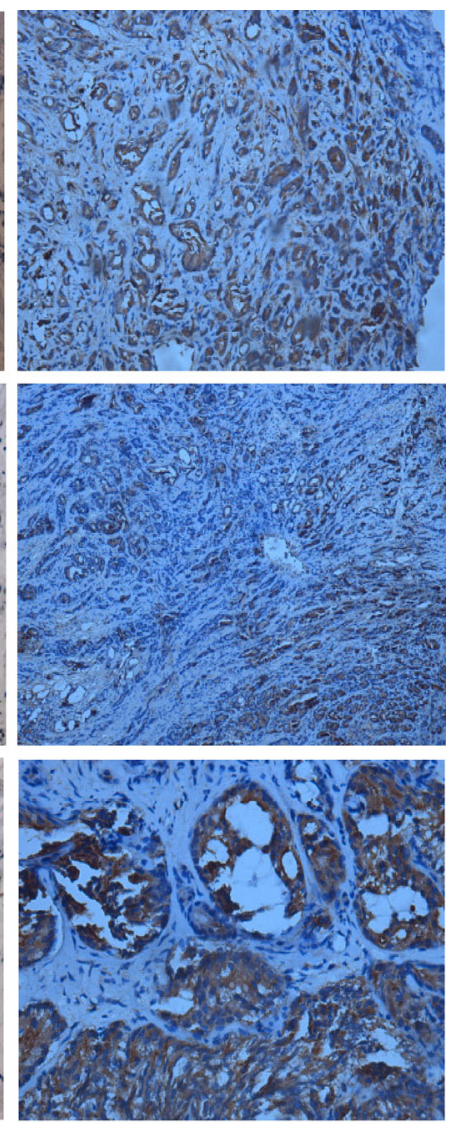

Fig. 1 TET1 is decreased in human hepatocellular cells. a RT-PCR detection of TET1 mRNA in normal and cancerous human cell lines. b Western blot detection of TET1 and TET3 protein level in normal and cancerous human cell lines. c IHC detection of TET1 protein in

The coding sequence of TET1 was cloned into the EcoRI/XhoI and BamHI/EcoRI sites of pc3-gab, respectively. The pc3-gab was produced based upon pcDNA3.0 (Invitrogen, Carlsbad, CA) by replacing the neomycin open reading frame with an expression cassette of the enhanced green fluorescent protein (EGFP) gene [21]. The generated expression vectors were named pc3-gab-TET1.

\section{Cell transfections}

Reverse transfection of RNA oligoribonucleotides was performed using Lipofectamine-RNAiMAX (Invitrogen). Transfection of plasmid DNA or co-transfection of RNA duplex with plasmid DNA was performed using Lipofectamine2000 (Invitrogen). Unless otherwise indicated, $50 \mathrm{nM}$ of RNA duplex and $200 \mathrm{nM}$ of miRNA inhibitor were used for each transfection. normal and cancerous human HCC tissues. Shown in right are three representative examples of normal adjacent tissues and tumor sections. Original magnification is 3,200 (normal) and 3,400 (cancer)

Table 2 Expression of TET1 detected with immunohistochemistry in human

\begin{tabular}{lll}
\hline & Non-tumoral liver tissues & Liver tumors \\
\hline Positive TET1 expression & 3 & 19 \\
Negative TET1 expression & 22 & 6 \\
\hline
\end{tabular}

Cell proliferation assay

Cell proliferation was monitored using Cell Counting Kit8 (CCK-8) (Dojindo, Japan). HepG2 and Huh-7 cells (3,000 per well) were transfected with miR-193b or siRNA against CCND1 or TET1 in 96-well culture plates. Seventy-two hours later, $5 \mu$ l CCK- 8 reagents was added to each well and incubated at $37^{\circ} \mathrm{C}$ for $1 \mathrm{~h}$. The number of viable cells was assessed by the measurement of absorbance at $450 \mathrm{~nm}$. 
Fig. 2 TET1 induces G1 arrest and inhibits growth in HepG2 and Huh-7 cells. a TET1 and siRNA against CCND1 triggered G1 arrest in HepG2 and Huh-7 cells. HepG2 and Huh-7 cells were treated with nocodazole $24 \mathrm{~h}$ post transfection, and cell cycle distribution was detected $20 \mathrm{~h}$ later. $2 \mathrm{~N}$, cells having diploid DNA content; $4 \mathrm{~N}$, cells having tetraploid DNA content. b G1 arrest could be induced by TET1 expressed from genomic fragment. HepG2 and Huh-7 cells were transfected with pcDNA3.0 and pcDNA-TET1. The cells were collected and used for cell cycle analysis $48 \mathrm{~h}$ post transfection. c-d TET1 inhibited growth in HepG2 and Huh-7 cells. The viable cells $72 \mathrm{~h}$ after transfection were detected by CCK-8 assay as described. Data are means of three separated experiments $\pm \mathrm{SD}, * * P<0.01$

\section{A}
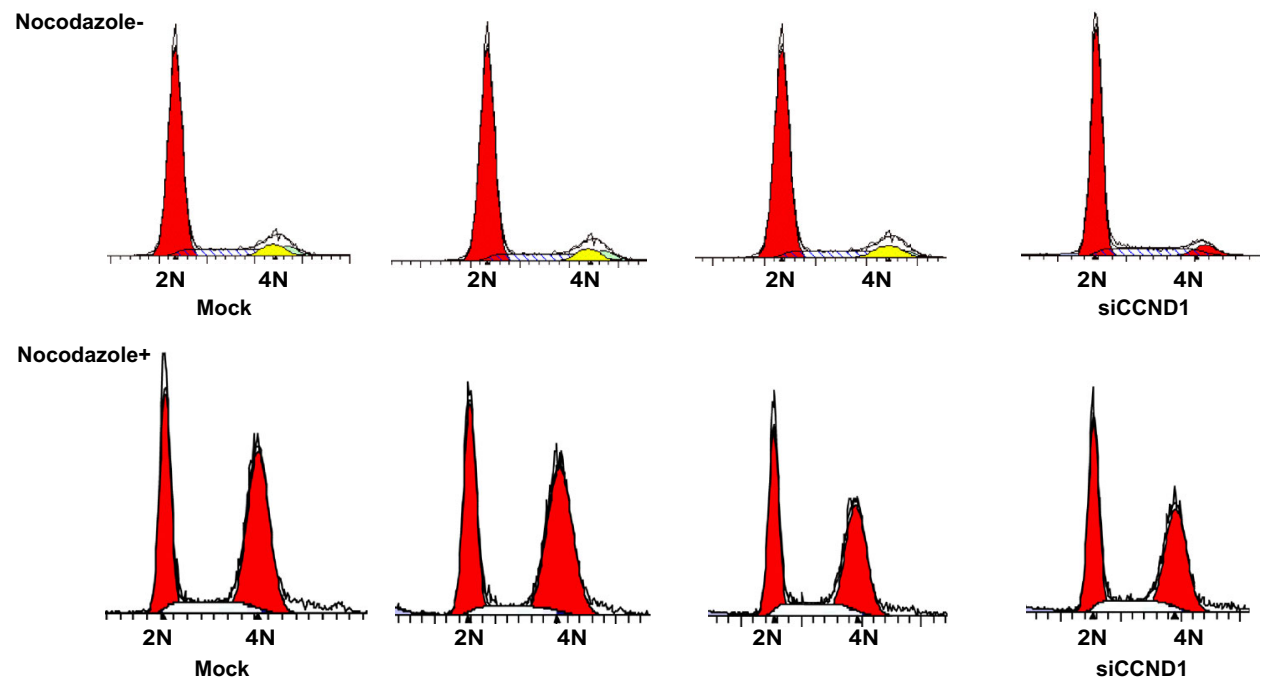

B
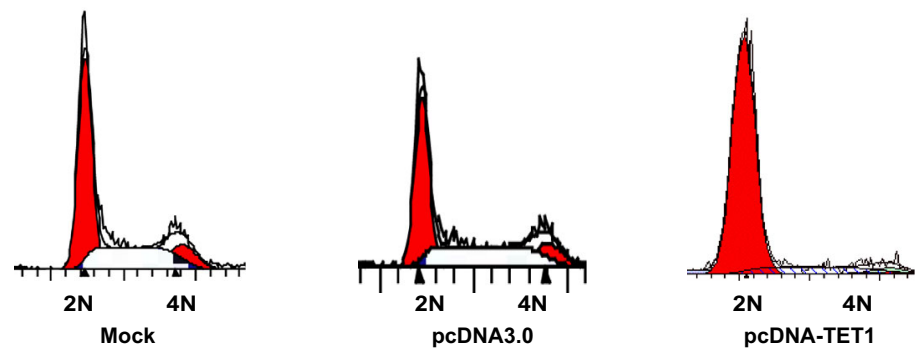

C

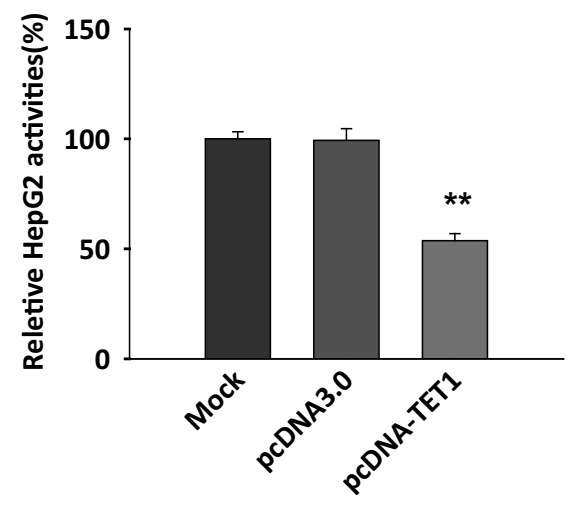

D

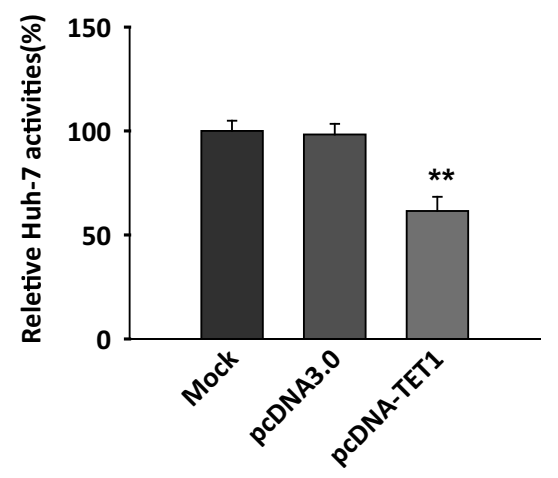

Transwell cell invasion and migration assay

For invasion assay, HepG2 and Huh-7 transfectants were serum starved for $24 \mathrm{~h}$ in DMEM containing $0.1 \%$ FBS. Serum-starved cells were trypsinized and resuspended in DMEM containing $0.1 \%$ FBS, and $2 \times 10^{5}$ cells were added to the upper chamber of each well $(6.5 \mathrm{~mm}$ in diameter, $8 \mathrm{~lm}$ pore size; Corning, NY, USA) coated with $30 \mathrm{mg} / \mathrm{cm}^{2}$ Matrigel (ECM gel, Sigma-Aldrich, St. Louis, $\mathrm{MO})$. Medium containing $0.1 \% \mathrm{FBS}$ and supplemented with
HGF (20 ng/ml) was placed in the lower compartment of the chamber. After $24 \mathrm{~h}$ at $37{ }^{\circ} \mathrm{C}$, cells on the upper membrane surface were removed by careful wiping with a cotton swab and the filters were fixed by treatment with $95 \%$ ethanol for $30 \mathrm{~min}$ and stained with $0.2 \%$ crystal violet solution for $30 \mathrm{~min}$. Invasive cells adhering to the undersurface of the filter were then counted (five high-power fields/chamber) using an inverted microscope. The migration assay is the same with invasion assay excepting that no Matrigel was used and the permeating time for cells was $12 \mathrm{~h}$. 


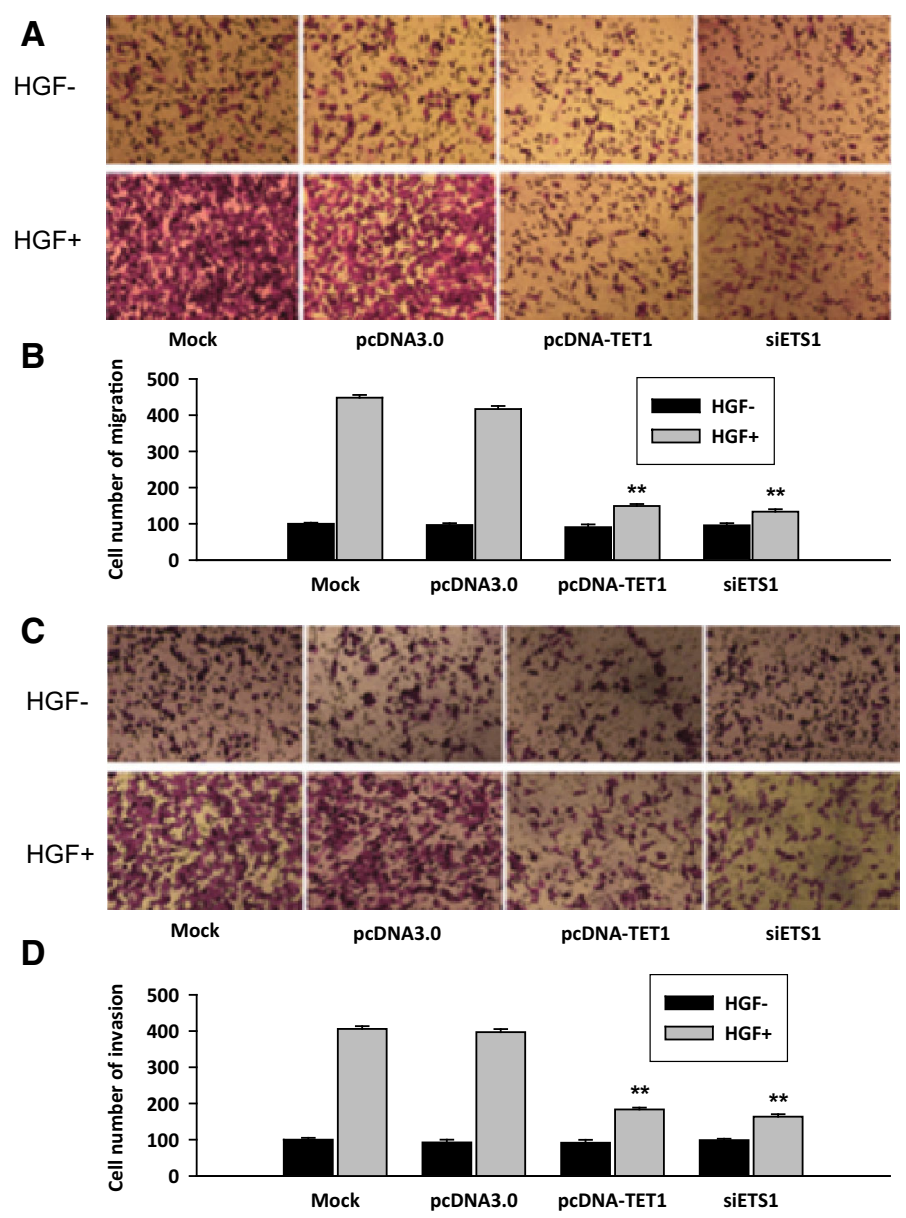

Fig. 3 TET1 suppresses migration and invasion of HepG2 and Huh-7 cells by repressing the expression of ETS1. a Cell migration assay. HepG2 cells were transfected with pcDNA-TET1 or ETS1 siRNA and then subjected to transwell migration assays, as described. After $12 \mathrm{~h}$, migration cells were counted after staining with crystal violet. b Migration cell numbers are the average count of three random microscopic fields. Each bar represents the mean \pm SD of the counts from a single representative experiment. ${ }^{*} P<0.01$. c Cell invasion

\section{Western blot}

Cells and ground tissues were lysed, equalized, loaded and blotted as described previously [29]. Antibodies specific to TET1, $\beta$-actin and horseradish-peroxidase-coupled secondary antibodies were purchased from Santa Cruz Biotechnology. Densitometric analysis was carried out with Lab-works Image Acquisition and Analysis software. The background was subtracted, and the signals of the detected bands were normalized to the amount of loading control $\beta$-actin band. The relative value was presented as fold increase over control sample as indicated.

\section{Statistical analysis of data}

Data are presented as mean \pm SD. Statistical comparisons between experimental groups were analyzed by Student's assay. Cells transfected with control RNA, pcDNA-TET1or ETS1 siRNA were subjected to transwell invasion assays, as described. After incubation for $24 \mathrm{~h}$, cells that invaded through the pores to the under surface of the membrane were fixed, stained and counted. d Invading cell numbers are the average count of three random microscopic fields. Each bar represents the mean \pm SD of the counts from a single representative experiment, $* * P<0.01$

$t$ test, and two-tailed $P<0.05$ was taken to indicate statistical significance.

\section{Results}

TET1 expression is downregulated in HCC

Given the established role of TET1 in DNA methylation in cancer, we investigated the role of TET1 in tumor development. In vitro, we detected TET1 mRNA with RT-PCR in normal and cancerous human cell lines (Fig. 1a), and the mRNA quantity is reduced in these tumor cells. TET1 and TET3 protein levels were detected by Western blot method, and TET protein expression trend is the same with mRNA (Fig. 1b). TET1 expression was analyzed in 17 paired HCC and adjacent non-tumor liver tissues by way of 
A
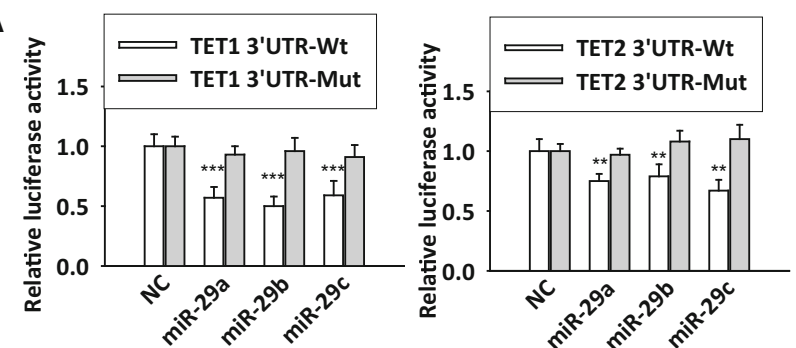

C
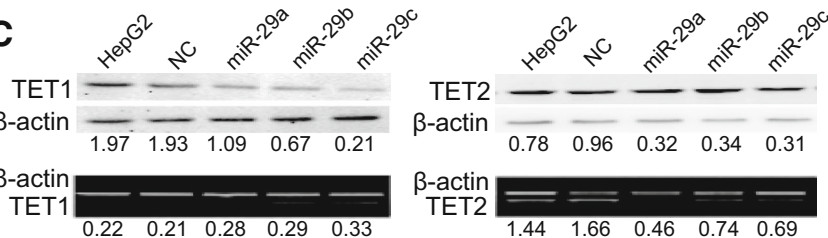

B

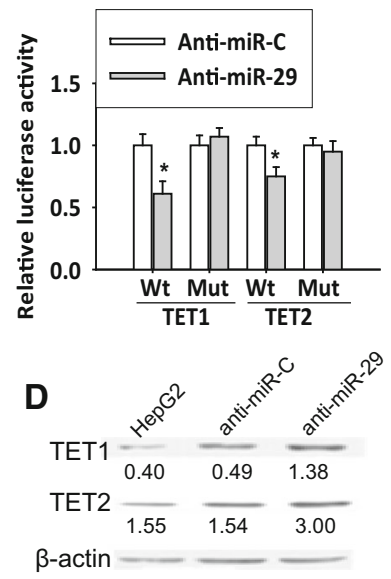

Fig. 4 TET1 is a direct target of miR-29. a, b Luciferase activity analysis, a cells were cotransfected with indicated RNA duplex, pRLTK and firefly luciferase reporter plasmid containing wild-type or mutant $3^{\prime}$-UTR of putative target gene. Cells were first transfected with anti-miR-29 or anti-miR-C and then with pRL-TK and the firefly luciferase reporter comprising the wild-type or mutant $3^{\prime}$-UTR of the putative target gene (b). pRL-TK expressing Renilla luciferase was cotransfected as an internal control to correct the differences in both transfection and harvest efficiencies. The firefly luciferase activity of each sample was normalized to the Renilla luciferase activity. The normalized luciferase activity of NC or anti-miR-C transfectant was set as relative luciferase activity; therefore, no error bar was shown

IHC detection. Immunohistochemical staining demonstrated that all liver cancer patients showed reduced TET1 expression in cancerous cells as compared to non-neoplastic glands (Fig. 1c). Note that the samples were diagnosed as liver cancer cells via $\mathrm{H} \& \mathrm{E}$ staining and stained with rabbit IgG as a negative control (data not shown). The results showed that TET1 was significantly repressed (2-330 fold) in $76 \%$ of tumors ( 19 of 25 patients) compared to the matching adjacent non-tumor liver tissues (Tables 1,2). These results suggest that downregulation of TET1 may be involved in most of human HCC development, as demonstrated in the following experiments.

TET1 represses cell proliferation by inducing a G1 arrest in HepG2 and Huh-7 cells

Repression of CCND1 prevents cells from entering the $\mathrm{S}$-phase, causing an accumulation of cells in G0/G1. To verify whether TET1 can trigger G1 arrest in hepatoma cell lines, TET1 and CCND1 siRNA against were transfected into HepG2 and Huh-7 cells. Mock-transfected HepG2 and Huh-7 cells showed a normal cell cycle distribution. In contrast, cell cultures transfected with pcDNA-TET1 or siCCND1 had increased numbers of cells in G0/G1 and corresponding decreases in the numbers of cells in $\mathrm{S}$ and G2/M (Fig. 2a). This finding suggests that TET1 can for NC and anti-miR-C transfectant. Values represent the mean \pm standard error of the mean of at least three independent experiments performed in duplicate. $* P<0.05 ; \quad * * P<0.01$, $* * * P<0.001$ compared with NC or anti-miR-C transfectants. Effects of miR-29 overexpression (c) or suppression (d) on the endogenous TET1 levels. Forty-eight hours after transfection with the indicated RNA oligo ribonucleotides, HepG2 cells were analyzed by Western blotting and RT-PCR. The intensity for each band was densitometrically quantified. The value under each lane indicates the relative expression level of the putative target gene, which is represented by the intensity ratio between TET1 and actin bands in each lane to controls. $* P<0.05$ relative to controls

induce G1 arrest in HepG2 and Huh-7 cell lines. The G0/ G1 accumulation phenotype became clear when the microtubule-depolymerizing drug was added $24 \mathrm{~h}$ post transfection to block cells from reentering the cell cycle after mitosis. This treatment caused most of the mocktransfected cells to accumulate in G2/M, whereas a large fraction of pcDNA-TET1 or siCCND1-transfected cells remained in G0/G1 (Fig. 2b). To confirm that the cellular phenotype induced by TET1 is not artificial, we determined whether G0/G1 cell accumulation phenotype could be induced by TET1 expressed from genomic fragment. We next evaluated the effect of TET1 on the growth of HCC cells. The results of cell proliferation assay showed that ectopic expression of TET1 led to significant inhibition of HepG2 and Huh-7 proliferation compared to control cells (Fig. 2c, d).

TET1 negatively regulates cell migration and invasion

Transcription factor ETS1 is over-expressed and plays a crucial role in the invasive property in hepatoma cell lines [30, 31]. HGF, also known as scatter factor, can induce the expression of ETS1 in hepatoma cells by binding with hepatocyte growth factor receptor (MET) [31]. Then, we examined the effect of TET1 on HGF-induced migration of HepG2 and Huh-7 cells using the transwell migration 

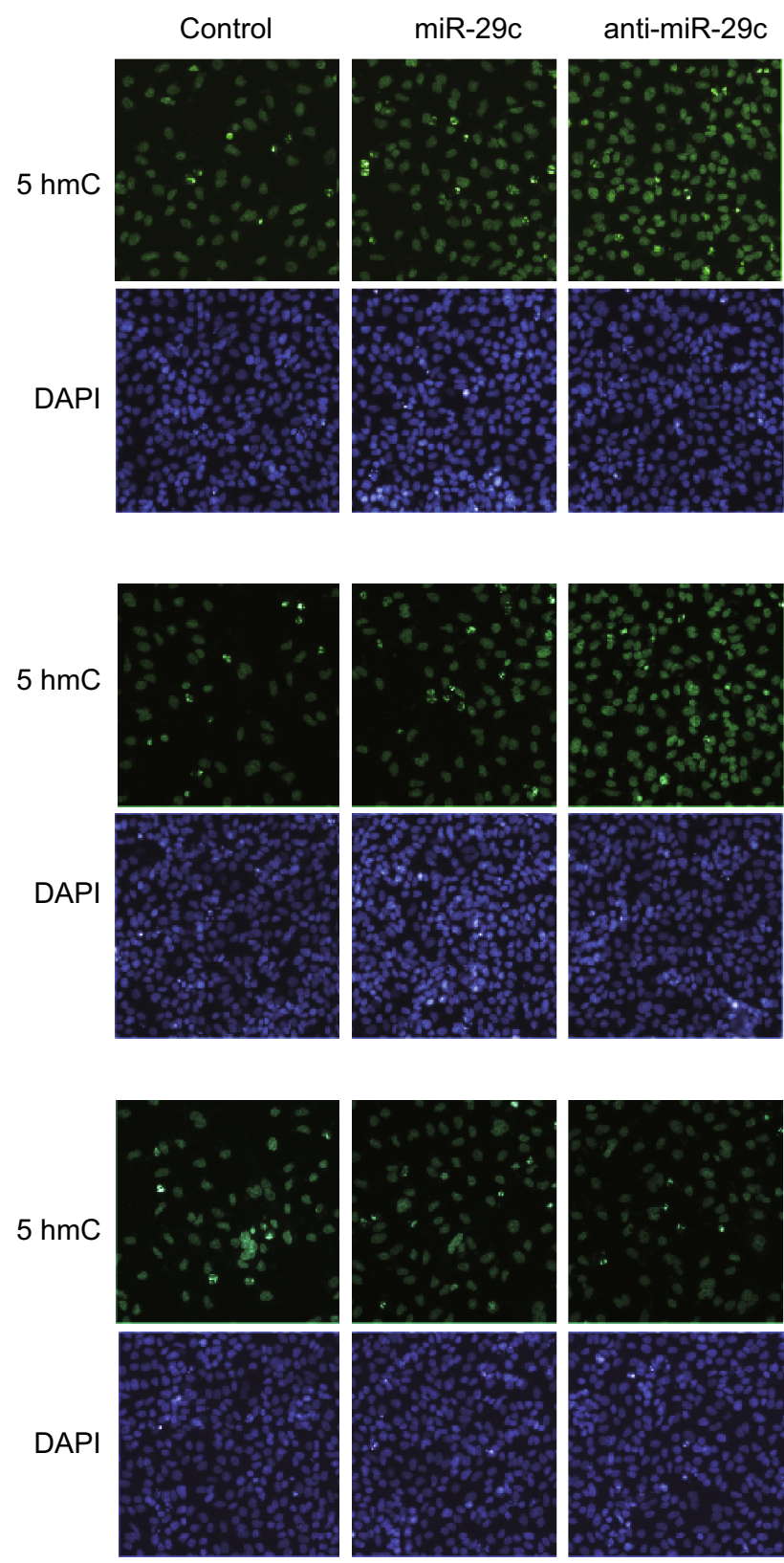

Fig. 5 miRNA-29-dependent regulation of $5 \mathrm{hmC}$ level. DAPI (blue) and $5 \mathrm{hmC}$ (green) were stained in HepG2 cells. HepG2 cells were transfected with pre-miR-29a, anti-miR-29a or control. After $48 \mathrm{~h}$, cells were fixed and immunofluorescence was performed

assay. The migration of HepG2 and Huh-7 cells was significantly induced by HGF, and TET1 could reduce the migration induced by HGF (Fig. 3a, b). Next, the effects of TET1 on the invasion of HepG2 and Huh-7 cells were determined by Matrigel invasion assay system. In line with the results from the above-mentioned migration assays, TET1 also inhibited the invasion of HepG2 and Huh-7 cells (Fig. 3c, d). To rule out other target effect of TET1 on cell migration and invasion, we used ETS1 siRNA to downregulate the ETS1 gene expression. siETS1 could also inhibit the migration and invasion induced by HGF (Fig. 3a-d). In addition, our results suggested that the reduction in the number of migrative/invasive cells is not due to apoptosis and inhibition of cell proliferation, and similar results were observed in HepG2 and Huh-7 cell lines (not shown). Taken together, the results mentioned above suggest that TET1 is a potent suppressor of hepatoma cell migration and invasion by repressing the expression of transcription factor ETS1, at least in partial.

TET are direct targets of miR-29

Next, we explored the molecular mechanisms responsible for the function of TET1 that were observed above. Predicted target genes of TET1 were retrieved using publicly available databases (Target Scan and miRanda). miR-29a/ $\mathrm{b} / \mathrm{c}$ was chosen for further analysis, because they are proapoptotic members of the microRNA family and have displayed frequent low-expression in HCC tissues. To verify whether TET1 is a direct target of miR-29, a dualluciferase reporter system was first employed. Co-transfection of $\mathrm{miR}-29 \mathrm{a} / \mathrm{b} / \mathrm{c}$ significantly increased the firefly luciferase activity of the reporter with wild-type $3^{\prime}$-UTR but not that of the mutant reporter (Fig. 4a). In addition, inhibition of endogenous miR-29a/b/c by anti-miR-29 led to reduced firefly luciferase activity of the wild-type reporter but not that of the mutant one (Fig. 4b). Further investigation showed that transfection with $\mathrm{miR}-29 \mathrm{a} / \mathrm{b} / \mathrm{c}$ increased the endogenous expression of under conditions of conventional culture (Fig. 4c). In addition, antagonism of endogenous miR-29 resulted in the downregulation of TET1 proteins (Fig. 4d).These data suggest that miR-29 may regulate the expression of TET1 by directly targeting the $3^{\prime}$-UTR of their mRNAs. Data of immunofluorescence show that $5 \mathrm{hmC}$, regulated by TET1, was also regulated by miR-29a (Fig. 5).

miRNA-29-dependent regulation of TET1 expression

Reduced expression of miR-29 is a frequent event in HCC and is correlated with a poor prognosis [13]. miR-29 can modulate the expression of DNMT-1 and 3B in leukemia and lung cancer cells $[25,32]$. We confirmed a similar miR-29a-dependent modulation of TET1 protein expression in HCC cells. As a result, we focused our attention on miR-29a, the isoform mainly represented in hepatocytes. The expression of miR-29a in several HCC cell lines and human HCC tissues correlated with the expression of TET1 with a $P$ value of 0.04 (Fig. 6a). Over-expression of miR29a in HCC cells decreased TET1 expression (Fig. 6b). All together, these studies suggest that miR-29 may indirectly modulate TET1 expression by acting on the methylation machinery. Our data suggest that the effects of 

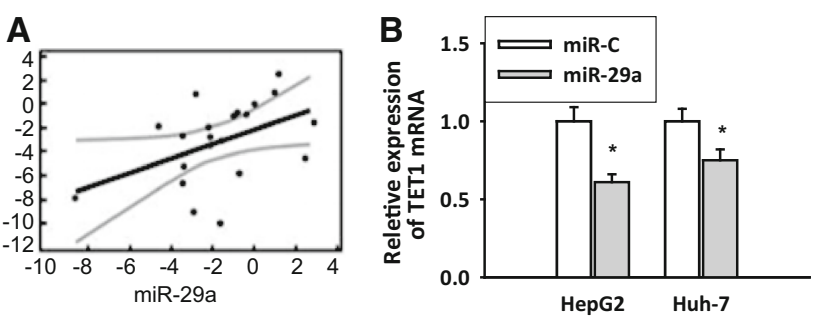

Fig. 6 miRNA-29-dependent regulation of TET1 expression a miR29a and TET1 expression was assessed in non-malignant human hepatocytes (HH), HCC cells (HepG2, Huh-7), human HCC tissues and adjacent non-tumoral tissues. miR-29a expression was assessed by TaqMan real-time PCR assay and normalized to that of RNU6B. TET1 expression was assessed by PCR using SYBR Green and normalized to that of GAPDH. In the graph, miR-29a and TET1 are expressed relative to $\mathrm{HH}$ for the cell lines or to adjacent tissues for human HCC (log scale). Regression analysis was performed using the MedCalc Software. The fitted regression line (black) and $95 \%$ confidence intervals (gray) are shown. (b) HepG2 and Huh7 cells were transfected with pre-miR-29a or control. After $48 \mathrm{~h}$, cells were collected, RNA extracted and real-time PCR performed. Bars represent mean and standard error of three independent experiments, relative to controls. $* P<0.05$ relative to controls

miR-29 on apoptosis may be at least in part mediated by the modulation of TET1.

\section{Discussion}

DNA methylation at the 5-position of cytosine (5-methylcytosine; $5 \mathrm{mC}$ ) is identified as one of the key epigenetic marks in the development and genome regulation [25, 33]. Studies have demonstrated that different genomic regions are differentially methylated based on cell or tissue type and developmental stage [34].

The enzymes that catalyze DNA methylation, known as the DNA methyl transferases (DNMTs), including DNMT1, DNMT3A, DNMT3B and the regulatory subunit DNMT3L, have already been identified and well characterized [35]. However, the differential and dynamical process regulated by genome-wide DNA methylation during 1 processes and the downstream gene expression which may be linked to DNA methylation remain unclear.

Recently, human ten-eleven translocation 1 (TET1), a member of the TET family of proteins, was identified responsible for catalyzing the conversion of $5 \mathrm{mC}-5 \mathrm{hmC}$. The role of TET proteins and $5 \mathrm{hmC}$ correlative diseases has been widely studied in the past 3 years since biochemical connection between TET proteins and $5 \mathrm{hmC}$ was firstly established. The mammalian TET family contains three members, TET1, TET2 and TET3, and each shares a high degree of homology within their C-terminal catalytic domain [36]. The identification of TET family of enzymes revealed a potentially molecular mechanism for the regulation of DNA methylation, indicating that $5 \mathrm{hmC}$ might act as an intermediate during the process of DNA demethylation. In our studies, we investigated expression levels and biological function of TET family enzymes in mouse cancer cells. It showed that TET1 and TET2, but not TET3, were expressed in cancer cells. Furthermore, TET1 and TET 2 could oxidize $5 \mathrm{mC}-5 \mathrm{hmC}$, adding complexity to uncovering the potential function of TET family enzymes and $5 \mathrm{hmC}$ in epigenetic regulation in cancer cells. The conditional knockdown of TET1 was used for further determination of biological function. The cancer cells with TET1 knockdown showed lower levels of 5hmc, which might contribute to aberrant DNA methylation patterns or other aspects in cancers. Moreover, the TET1 knockdown resulted in the growth stimulation and inhibition of cell apoptosis, indicating that TET1 is an important regulator and TET1 mutations or dysregulation might lead to lethality.

Recently, it has been shown that $\mathrm{miR}-29 \mathrm{a} / \mathrm{b} / \mathrm{c}$ upregulates p53 by targeting p 85 and CDC 42 and induces apoptosis in a p53-dependent manner in breast and colorectal cancer cell lines [26]. miR-29b is also shown to target Mcl1 and sensitize cholangio carcinoma cells to tumor necrosis factor-related apoptosis-inducing ligand-induced apoptosis [26]. In the present study, we employed a new research model, HCC cells, to study the apoptosis pathways by which miR-29 exerts its function and modulates the malignant phenotypes of cancer cells. We believed that TET1 is a target of miR-29. This study, together with the work of other groups, demonstrates that miR-29 may target multiple proteins that function spatiotemporally or in cooperation in different cellular processes including cell growth, death and differentiation [24, 25, 29]. It is intriguing that miRNA can suppress multiple genes that favor the process of tumorigenesis, because intake of a single miRNA may modulate complex downstream signals and inhibit tumor growth. Hence, miRNA is more likely to be an effective as anticancer drugs.

Our data suggest that functional loss of miR-29 family may result in enhanced expression of TET1 during the resistance of cells to apoptosis, which consequently favors tumor progression. We are aware that other miR-29 target genes may also be involved in miR-29 promoted apoptosis. However, we observed that silencing of TET1 can largely mimic the apoptosis-promoting effect of miR-29 overexpression and TET 1 expression can dramatically reverse the effect of miR-29, which implicates TET1 as predominant mediators of miR-29-promoted apoptosis in HCC cells.

In summary, we investigated the potential role of feedback of TET1-miR-29 family in tumorigenesis and its underlying mechanisms. Our data suggested that downregulation of TET1 play an important role in the development of cancer, such as HCC, and that miR-29 be 
employed as prognosis marker and therapeutic target for HCC.

Acknowledgments This study was supported by Chinese Foundation for Hepatitis Prevention and Control project (20120030) and the Medical research projects of Wuxi Hospital Management Center (ygzxl 1306).

Conflict of interest The authors declare no conflict of interest.

\section{References}

1. Aravalli RN, Steer CJ, Cressman EN. Molecular mechanisms of hepatocellular carcinoma. Hepatology. 2008;48(6):2047-63.

2. Villanueva A, Toffanin S, Llovet JM. Linking molecular classification of hepatocellular carcinoma and personalized medicine: preliminary steps. Curr Opin Oncol. 2008;20(4):444-53.

3. Lorsbach RB, Moore J, Mathew S, Raimondi SC, Mukatira ST, Downing JR. TET1, a member of a novel protein family, is fused to MLL in acute myeloid leukemia containing the $\mathrm{t}(10 ; 11)(\mathrm{q} 22 ; \mathrm{q} 23)$. Leukemia. 2003;17(3):637-41.

4. Hsu CH, Peng KL, Kang ML, Chen YR, Yang YC, Tsai CH, et al. TET1 suppresses cancer invasion by activating the tissue inhibitors of metalloproteinases. Cell Rep. 2012;2(3):568-79.

5. Albano F, Anelli L, Zagaria A, Coccaro N, Minervini A, Rossi $\mathrm{AR}$, et al. Decreased TET2 gene expression during chronic myeloid leukemia progression. Leuk Res. 2011;35(11):e220-2.

6. Euba B, Vizmanos JL, Garcia-Granero M, Aranaz P, Hurtado C, Migueliz I, et al. A meta-analysis of TET2 mutations shows a distinct distribution pattern in de novo acute myeloid leukemia and chronic myelomonocytic leukemia. Leuk Lymphoma. 2012;53(6):1230-3.

7. Figueroa ME, Abdel-Wahab O, Lu C, Ward PS, Patel J, Shih A, et al. Leukemic IDH1 and IDH2 mutations result in a hypermethylation phenotype, disrupt TET2 function, and impair hematopoietic differentiation. Cancer Cell. 2010;18(6):553-67.

8. Ko M, Huang Y, Jankowska AM, Pape UJ, Tahiliani M, Bandukwala HS, et al. Impaired hydroxylation of 5-methylcytosine in myeloid cancers with mutant TET2. Nature. 2010;468(7325):839-43.

9. Bushati N, Cohen SM. microRNA functions. Annu Rev Cell Dev Biol. 2007;23:175-205.

10. Spizzo R, Nicoloso MS, Croce CM, Calin GA. SnapShot: MicroRNAs in cancer. Cell. 2009;137(3):586e1.

11. Garzon R, Calin GA, Croce CM. MicroRNAs in cancer. Annu Rev Med. 2009;60:167-79.

12. Ji J, Shi J, Budhu A, Yu Z, Forgues M, Roessler S, et al. MicroRNA expression, survival, and response to interferon in liver cancer. N Engl J Med. 2009;361(15):1437-47.

13. Xiong Y, Fang JH, Yun JP, Yang J, Zhang Y, Jia WH, et al. Effects of microRNA-29 on apoptosis, tumorigenicity, and prognosis of hepatocellular carcinoma. Hepatology. 2010;51(3):836-45.

14. Braconi C, Valeri N, Gasparini P, Huang N, Taccioli C, Nuovo G, et al. Hepatitis $\mathrm{C}$ virus proteins modulate microRNA expression and chemosensitivity in malignant hepatocytes. Clin Cancer Res. 2010;16(3):957-66.

15. Pineau P, Volinia S, McJunkin K, Marchio A, Battiston C, Terris B, et al. miR-221 overexpression contributes to liver tumorigenesis. Proc Natl Acad Sci USA. 2010;107(1):264-9.

16. Jiang J, Gusev Y, Aderca I, Mettler TA, Nagorney DM, Brackett DJ, et al. Association of MicroRNA expression in hepatocellular carcinomas with hepatitis infection, cirrhosis, and patient survival. Clin Cancer Res. 2008;14(2):419-27.

17. Meng F, Henson R, Wehbe-Janek H, Ghoshal K, Jacob ST, Patel T. MicroRNA-21 regulates expression of the PTEN tumor suppressor gene in human hepatocellular cancer. Gastroenterology. 2007;133(2):647-58.

18. Gramantieri L, Ferracin M, Fornari F, Veronese A, Sabbioni S, Liu CG, et al. Cyclin G1 is a target of miR-122a, a microRNA frequently down-regulated in human hepatocellular carcinoma. Cancer Res. 2007;67(13):6092-9.

19. Wong QW, Lung RW, Law PT, Lai PB, Chan KY, To KF, et al. MicroRNA-223 is commonly repressed in hepatocellular carcinoma and potentiates expression of Stathmin1. Gastroenterology. 2008;135(1):257-69.

20. Wang Y, Lee AT, Ma JZ, Wang J, Ren J, Yang Y, et al. Profiling microRNA expression in hepatocellular carcinoma reveals microRNA-224 up-regulation and apoptosis inhibitor-5 as a microRNA-224-specific target. J Biol Chem. 2008;283(19): 13205-15.

21. Su H, Yang JR, Xu T, Huang J, Xu L, Yuan Y, et al. MicroRNA101, down-regulated in hepatocellular carcinoma, promotes apoptosis and suppresses tumorigenicity. Cancer Res. 2009;69(3): $1135-42$.

22. Xu T, Zhu Y, Xiong Y, Ge YY, Yun JP, Zhuang SM. MicroRNA195 suppresses tumorigenicity and regulates G1/S transition of human hepatocellular carcinoma cells. Hepatology. 2009;50(1): 113-21.

23. Law PT, Wong N. Emerging roles of microRNA in the intracellular signaling networks of hepatocellular carcinoma. J Gastroenterol Hepatol. 2011;26(3):437-49.

24. Wang H, Garzon R, Sun H, Ladner KJ, Singh R, Dahlman J, et al. NF-kappaB-YY1-miR-29 regulatory circuitry in skeletal myogenesis and rhabdomyosarcoma. Cancer Cell. 2008;14(5): 369-81.

25. Fabbri M, Garzon R, Cimmino A, Liu Z, Zanesi N, Callegari E, et al. MicroRNA-29 family reverts aberrant methylation in lung cancer by targeting DNA methyltransferases $3 \mathrm{~A}$ and $3 \mathrm{~B}$. Proc Natl Acad Sci USA. 2007;104(40):15805-10.

26. Mott JL, Kobayashi S, Bronk SF, Gores GJ. mir-29 regulates Mcl-1 protein expression and apoptosis. Oncogene. 2007;26(42):6133-40.

27. Braconi C, Kogure T, Valeri N, Huang N, Nuovo G, Costinean S, et al. microRNA-29 can regulate expression of the long noncoding RNA gene MEG3 in hepatocellular cancer. Oncogene. 2011;30(47):4750-6.

28. Pekarsky Y, Santanam U, Cimmino A, Palamarchuk A, Efanov A, Maximov V, et al. Tcl1 expression in chronic lymphocytic leukemia is regulated by miR-29 and miR-181. Cancer Res. 2006;66(24):11590-3.

29. Park SY, Lee JH, Ha M, Nam JW, Kim VN. miR-29 miRNAs activate p53 by targeting p85 alpha and CDC42. Nat Struct Mol Biol. 2009;16(1):23-9.

30. Ozaki I, Mizuta T, Zhao G, Yotsumoto H, Hara T, Kajihara S, et al. Involvement of the Ets-1 gene in overexpression of matrilysin in human hepatocellular carcinoma. Cancer Res. 2000;60(22):6519-25.

31. Jiang $\mathrm{Y}, \mathrm{Xu} \mathrm{W}, \mathrm{Lu} \mathrm{J}, \mathrm{He} F$, Yang X. Invasiveness of hepatocellular carcinoma cell lines: contribution of hepatocyte growth factor, c-met, and transcription factor Ets-1. Biochem Biophys Res Commun. 2001;286(5):1123-30.

32. Garzon R, Liu S, Fabbri M, Liu Z, Heaphy CE, Callegari E, et al. MicroRNA-29b induces global DNA hypomethylation and tumor suppressor gene reexpression in acute myeloid leukemia by targeting directly DNMT3A and 3B and indirectly DNMT1. Blood. 2009;113(25):6411-8. 
33. Cedar H, Bergman Y. Linking DNA methylation and histone modification: patterns and paradigms. Nat Rev Genet. 2009;10(5):295-304.

34. Bestor T, Laudano A, Mattaliano R, Ingram V. Cloning and sequencing of a cDNA encoding DNA methyltransferase of mouse cells. The carboxyl-terminal domain of the mammalian enzymes is related to bacterial restriction methyltransferases. J Mol Biol. 1988;203(4):971-83.
35. Bourc'his D, Xu GL, Lin CS, Bollman B, Bestor TH. Dnmt3L and the establishment of maternal genomic imprints. Science. 2001;294(5551):2536-9.

36. Loenarz C, Schofield CJ. Oxygenase catalyzed 5-methylcytosine hydroxylation. Chem Biol. 2009;16(6):580-3. 